\title{
Depressive Erkrankungen immer noch unterschätzt
}

$\mathrm{D}$ epressive Erkrankungen sind ein zunehmender Grund für Fehltage, Arbeitsunfähigkeit und Frühberentungen, wie gerade eine aktuelle Untersuchung der deutschen Rentenversicherungsträger und der Betriebskrankenkassen bestätigte. Rund acht Millionen Menschen leiden in Deutschland an Depressionen. Dennoch wird die gesundheitspolitische Brisanz dieses Thema immer noch unterschätzt. Auch die Versorgung der Patienten ist bislang unzureichend, obwohl mit Antidepressiva und Psychotherapie wirksame Behandlungsoptionen zur Verfügung stehen. Schätzungen gehen davon aus, dass lediglich $10 \%$ der behandlungsbedürftigen Patienten tatsächlich eine adäquate Akutbehandlung erhalten. Im Langzeitverlauf sieht die Situation noch problematischer aus.

Einer der Hauptgründe für diese Unterversorgung liegt sicherlich in der schwierigen Diagnosesituation. Depressive Patienten suchen nur in Ausnahmefällen direkt den Facharzt auf, die meisten klagen auch im Gespräch mit dem Hausarzt nur selten über depressive Symptome, im Vordergrund stehen körperliche Beschwerden und komorbide Erkrankungen. Darüber hinaus hat die Diskussion der letzten Monate um Wirksamkeit bzw. Unwirksamkeit der Antidepressiva bei Kindern sowie Berichte über suizidinduzierende Tendenzen zu Verunsicherung geführt.

In dieser Ausgabe der psychoneuro liegt ein Schwerpunkt daher auf der Komorbidität bei depressiven Erkrankungen. Am Beispiel der kardiovaskulären Erkrankungen stellen Agelink et al. Inzidenz, Ursachen und Folgen dieser komorbiden Erkrankung vor. Neben genetischen Faktoren werden endokrine, neurokardiologische, metabolische und behaviorale Faktoren diskutiert. In Anbetracht der signifikanten Interaktion zwischen kardiovaskulären und depressiven Erkrankungen, die zu den häufigsten Diagnosen zählen, ist eine engmaschige Vernetzung aller am Behandlungsprozess beteiligten Arztgruppen nicht zuletzt auch aus ökonomischen Gründen zur Vermeidung von Folgekosten zwingend notwendig.

Ein weiterer Grund für die Unterversorgung depressiver Patienten ist die unzureichende Langzeitbehandlung. Wie Wolfersdorf und Heindl in ihrem Beitrag erläutern, darf sich die Depressionsbehandlung heute nicht nur auf eine Symptombesserung in der Akutphase beschränken. Das Risiko für Chronifizierung und Wiedererkrankung kann durch rechtzeitige und adäquate Behandlung gesenkt werden. Aber auch chronische
Depressionen sind kein hoffnungsloses Unterfangen. Darüber hinaus beschreiben die beiden Autoren die verschiedenen klinischen Bilder der „langanhaltenden Depression“: primäre chronische Depression, Dysthymia (neurotische Depression), depressive Episode mit überdauernder Restsymptomatik und häufig rezidivierende depressive Episoden mit depressionsfreien Intervallen unter sechs Monaten.

Im Brennpunkt dieser Ausgabe geht Ulrich Hegerl der Frage nach, ob tatsächlich ein Zusammenhang zwischen Antidepressiva und Suizidalität besteht. Wie bei jeder Therapie ist auch bei der Behandlung einer schweren depressiven Episode mit Antidepressiva besondere Aufmerksamkeit notwendig bis die Wirksamkeit für den Patienten spürbar wird. Spekulationen, dass Antidepressiva, insbesondere die SSRI, in besonderem Maße die Gefahr der Suizidinduktion bergen, können aber, wie Hegerl vorstellt, weitgehend ausgeschlossen werden.

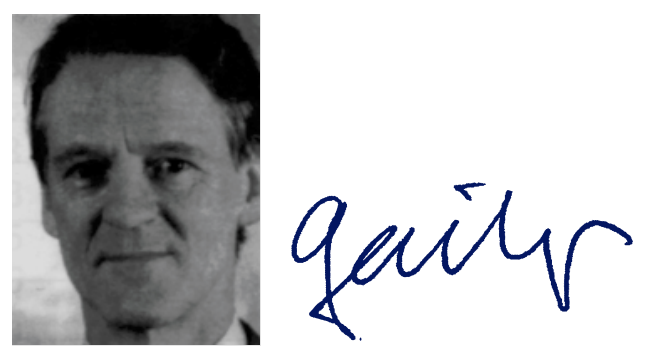

Prof. Dr. med. Markus Gastpar, Essen 